\title{
FUTEBOL Control Framework: Enabling Experimentation in Convergent Optical, Wireless, and Cloud Infrastructures
}

\author{
Cristiano Both, Rafael Guimaraes, Frank Slyne, Juliano Wickboldt, Magnos Martinello, Cristina Dominicini, \\ Rafael Martins, Yi Zhang, Diego Cardoso, Rodolfo Villaca, Isabella Ceravolo, Reza Nejabati, \\ Johann Marquez-Barja, Marco Ruffini, and Luiz DaSilva
}

\begin{abstract}
Large-scale testing and evaluation of network solutions are complex and typically involve multiple domains (e.g., optical, wireless, and cloud). The FUTEBOL project has deployed geographically distributed testbeds in Brazil and Europe that enable the experimentation and validation of new cross-domain network solutions. In this article, we introduce a Control Framework that allows experimenters to slice, reserve, and orchestrate optical, wireless, and cloud resources in a coordinated manner. We illustrate the features of our Control Framework and evaluate it through an experiment involving resource orchestration and automatic service scaling across multiple domains.
\end{abstract}

Index Terms-Cloud, Convergence, Optical, Testbed, Wireless

\section{INTRODUCTION}

Emerging telecommunication trends, such as cell densification, millimeter-wave, heterogeneous networks, and deviceto-device communications, pose stringent throughput, latency, energy, and cost requirements that demand the joint consideration of optical networks, wireless networks, and cloud architectures [1]. In this context, the design of next-generation networks requires experimental facilities that can offer realistic and at-scale environments for development, deployment, and validation. These facilities must offer an integrated control framework to virtualize and manage heterogeneous resources across different network infrastructures.

Such an integrated control framework must orchestrate heterogeneous network resources that sit across separate domains: time, frequency, wavelength, and space [2]. Besides, the heterogeneity of converged optical and wireless networks requires a high level of virtualization to decouple the software from the underlying hardware, by abstracting physical resources. While some investigations have recently addressed issues revolving around the convergence of optical and wireless networks, many experimental studies still address each domain separately [3]. Moreover, the existing control frameworks [4], [5] do not support experiments with full control of resources across the optical, wireless, and cloud boundaries [2].

Cristiano Both is with the University of Vale do Rio dos Sinos; Juliano Wickboldt, Rafael Martins are with the Federal University of Rio Grande do Sul (UFRGS); Rafael Guimaraes, Magnos Martinello, Cristina Dominicini, Diego Cardoso, Isabella Ceravolo, Rodolfo Villaca, are with the Federal University of Espirito Santos (UFES); Frank Slyne, Yi Zhang, Marco Ruffini, Luiz DaSilva are with Trinity College Dublin (TCD); Rafael Guimaraes, Reza Nejabati are with the University of Bristol (UNIVBRIS); Johann MarquezBarja is with the University of Antwerp \& imec.
To address these challenges, the Federated Union of Telecommunications Research Facilities for an EU-Brazil Open Laboratory (FUTEBOL) project [6] has established research infrastructure to enable experimental cross-domain network research, and developed a Control Framework as a suite of software components that provide management, control, and measurement tools to testbed users and operators. Our main contribution is the design and implementation of a Control Framework that bridges the gap between optical and wireless networks, linking them to the cloud, enabling the control of experiments across all three domains.

To support rich experiment scenarios (e.g., Internet of Things (IoT), Cloud Radio Access Network (C-RAN) and other 5G use cases), the FUTEBOL Control Framework builds on Network Functions Virtualization (NFV), SoftwareDefined Networking (SDN), and Software-Defined Radio (SDR) paradigms, allowing integrated control of optical, wireless, and computational resources. Our Control Framework provides unified optical-wireless-cloud orchestration, including: (i) cloud and NFV orchestration capabilities, such as policy-based automatic scaling of a Virtual Network Function (VNF), resource monitoring across testbeds based on container virtualization, and VNF life-cycle management; and (ii) integrated convergent network orchestration that interacts with the multiple network controllers in the wireless and optical domains that virtualize physical network resources.

We illustrate the functionality offered by the FUTEBOL Control Framework through an end-to-end experiment, in which resources ranging from physical to application layer must be orchestrated. Firstly, we evaluate the impact of scaling physical resources of optical and wireless access networks demanded of mobile users on an LTE network. Secondly, we analyze the effects of dynamically allocating computational resources using a cloud auto-scaling feature that adjusts the available resources according to the demands from a mobile application to a Web service. Our results show the impact of cross-layer orchestration on service performance, and demonstrate how networking solutions can be tested using the FUTEBOL Control Framework.

\section{RESEARCh INFRASTRUCTURE FOR NETWORKING EXPERIMENTATION}

This section discusses some of the most significant projects that have developed experimental facilities for networking 
TABLE I: Research projects for networking experimentation

\begin{tabular}{|c|c|c|c|c|c|}
\hline \multirow{2}{*}{ Project } & \multirow{2}{*}{ Objective } & \multicolumn{2}{|c|}{ Main Tools } & \multicolumn{2}{|c|}{ Orchestration and Programmability } \\
\hline & & Management & Slicing & Physical Layer & Link Layer \\
\hline $\begin{array}{l}\text { GENI: Global Environment for Net- } \\
\text { work Innovations }\end{array}$ & $\begin{array}{l}\text { Provide a virtual lab- } \\
\text { oratory for network- } \\
\text { ing }\end{array}$ & GCF & $\begin{array}{l}\text { FlowVisor; OpenVir- } \\
\text { teX FlowSpace Fire- } \\
\text { wall }\end{array}$ & $\begin{array}{l}\text { Optical } \\
\text { wavelengths }\end{array}$ & $\begin{array}{l}\text { Switch packet; Flows } \\
\text { Handover }\end{array}$ \\
\hline $\begin{array}{l}\text { OFELIA: Open Flow in Europe - Link- } \\
\text { ing Infrastructure and Applications }\end{array}$ & $\begin{array}{l}\text { OpenFlow-based to } \\
\text { control the network } \\
\text { environment }\end{array}$ & OCF & $\begin{array}{l}\text { Optical FlowVisor } \\
\text { VerTIGO }\end{array}$ & $\begin{array}{l}\text { Optical } \\
\text { wavelengths }\end{array}$ & Optical ports; Flows \\
\hline $\begin{array}{l}\text { OF@TEIN: } \text { OpenFlow/OpenFeder- } \\
\text { ation at Trans-Eurasia Information } \\
\text { Network }\end{array}$ & $\begin{array}{l}\text { OpenFlow-based } \\
\text { SDN }\end{array}$ & OCF & $\begin{array}{l}\text { FlowVisor; VLAN- } \\
\text { based }\end{array}$ & Optical switching & Switch packet; Flows \\
\hline $\begin{array}{l}\text { RISE: Research Infrastructure for } \\
\text { large-Scale Experiments }\end{array}$ & $\begin{array}{l}\text { OpenFlow infrastruc- } \\
\text { ture }\end{array}$ & $\begin{array}{l}\text { RISE Or- } \\
\text { chestrator }\end{array}$ & MAC Rewriting & None & $\begin{array}{l}\text { Switch Port \& Packet; } \\
\text { Flows }\end{array}$ \\
\hline $\begin{array}{l}\text { FIBRE: Future Internet Brazilian En- } \\
\text { vironment for Experimentation }\end{array}$ & $\begin{array}{l}\text { Future } \quad \text { Internet } \\
\text { testbed }\end{array}$ & OMF6 & FlowVisor; TDD & Wireless channels & $\begin{array}{l}\text { Switch Port \& Packet; } \\
\text { Flows }\end{array}$ \\
\hline $\begin{array}{l}\text { FUTEBOL: Federated Union of } \\
\text { Telecommunications Research Facili- } \\
\text { ties for an EU-Brazil Open Laboratory }\end{array}$ & $\begin{array}{l}\text { Optical, wireless, and } \\
\text { cloud convergence }\end{array}$ & $\begin{array}{l}\text { CBTM; } \\
\text { FOAM; } \\
\text { O2CMF }\end{array}$ & $\begin{array}{l}\text { TDD; Optical wave- } \\
\text { lengths Virtual In- } \\
\text { frastructure }\end{array}$ & \begin{tabular}{l}
\multicolumn{3}{l}{ Optical-Wireless } \\
Channels and \\
Bandwidth
\end{tabular} & $\begin{array}{l}\text { Switch Port \& Packet; } \\
\text { Flows; Handover }\end{array}$ \\
\hline
\end{tabular}

experimentation. In the United States, GENI established an open infrastructure for at-scale networking and distributed systems research and education [7]. In Europe, OFELIA created an experimental facility that uses OpenFlow to control the network environment [7]. The OF@TEIN project was designed to deploy a shared OpenFlow-based SDN testbed infrastructure [7]. Similarly, Japan has the RISE project, which offers OpenFlow-based research infrastructure on the Japan Gigabit Network [7]. In Brazil, the FIBRE project built federated testbeds for research and education. FUTEBOL was conceived to enable experimental research on optical, wireless, and cloud convergence [8]. The objectives and main tools employed by each project are summarized in Table I, and further discussed throughout this section.

The aforementioned projects use a set of Control Frameworks to: (i) manage heterogeneous physical resources, (ii) support a federated Application Programming Interface (API) for uniform access to allocated resources, and (iii) provide orchestration tools for experiment control. GENI designed their Control Framework to provide an open reference implementation of the Slice-based Federation Architecture (SFA). The GENI Control Framework relies on the Aggregate Manager (AM) component for resource instantiation, and on the Clearinghouse component for authorization [4]. Specifically, the OpenFlow Aggregate Manager (FOAM) is used by sites in GENI to allocate OpenFlow resources. Likewise, OFELIA created their Control Framework where users interact with the Clearinghouse and the AM through a web portal. As shown in Table I, some projects continue to use the OFELIA Control Framework, even though its development has been discontinued. In contrast, FIBRE uses the Control and Management Framework (OMF) V6, which supports wired and wireless devices [9].

In FUTEBOL, we have extended existing management tools, based on the GENI Control Framework, to fulfill the requirement of provisioning optical, wireless, and cloud resources, via Federation for Future Internet Research and Experimentation (Fed4FIRE). For provisioning wireless and optical resources, we have extended Cloud-Based Testbed Manager (CBTM) and FOAM, respectively, and have de- veloped OpenStack and OpenFlow Control and Management Framework (O2CMF) for cloud resources provisioning. Resources provisioned in different domains can be configured in an integrated manner during the orchestration phase, enabling convergent experiments.

Networking experiments require slicing support for sharing and isolating physical network resources. Slicing tools sit between the physical and control planes in a virtualization layer, enabling multiple control of the same forwarding elements to support multi-tenancy. In this context, GENI adopts OpenFlow FlowVisor, FlowSpace Firewall, and OpenVirteX to slice the substrate network. OFELIA chooses ViRtual TopologIes Generalization in OpenFlow networks (VeRTIGO) and Optical FlowVisor, while RISE slices its network by the logical path with Media Access Control (MAC) rewriting. OF@TEIN and FIBRE adopt FlowVisor for slicing. In contrast to previous projects, FUTEBOL provides networking slicing by offering a policy-based virtual infrastructure, which supports the Fed4FIRE API on top of a testbed cloud manager based on OpenStack. Moreover, FUTEBOL deals with the network convergence challenge by $(i)$ enabling time-domain scheduling for slicing of wireless channels; and (ii) supporting the assignment of a different wavelength to each experiment's slice.

Experimental infrastructure should provide flexible configuration through orchestration and programmability. The analysis summarized in Table I reflects that FUTEBOL differs from all other projects through its integrated orchestration of wireless, optical, packet, and cloud network resources. Besides, FUTEBOL extends the range of configurable physical layer (e.g., wireless and optical channels with adaptive bandwidth) and link layer (e.g., switch ports and packet, flows, and wireless handover) parameters that are exposed to network controllers. In this manner, it allows deep programmability for experimenters interested in controlling physical and link layer parameters of different domains. In the next section, we present the FUTEBOL Control Framework architecture and tools to enable optical, wireless, and cloud convergence. 


\section{CONTROL FRAMEWORK}

The FUTEBOL Control Framework (Fig. 1) allows experimenters to easily reserve resources and deploy complex experiments across multiple network domains, including resources from testbeds in Europe and Brazil. The components of the architecture reside within separate layers, which are designed for minimal inter-layer dependency. The separation of functionalities and components distinguishes among: $(i)$ Converged Physical Infrastructure Layer (optical, wireless, and cloud resources); (ii) Virtualization Layer; (iii) Experiment Control and Orchestration Layer; (iv) Service Layer; and (v) Testbed Management Layer. Each layer relates to an SFA building block [4].

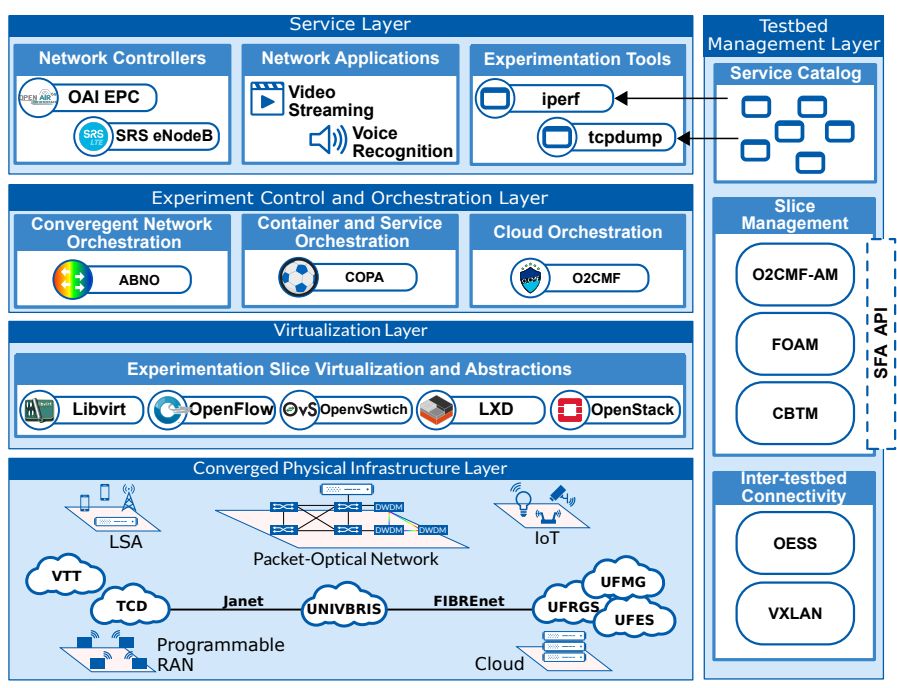

Fig. 1: FUTEBOL Control Framework Architecture

The Converged Physical Infrastructure Layer provides physical connectivity over wireless and optical network segments. The Control Framework has been deployed across several network testbeds in Europe and Brazil. In TCD, a programmable RAN is connected to computing servers through a Passive Optical Network (PON), which can emulate fronthaul/backhaul RAN scenarios supported by optical access networks e.g., Fiber To The X. The University of Bristol has an open and independent reconfigurable optical add/drop multiplexer with bandwidth-variable transceivers that is SDN-enabled for its optical ports. These ports are connected to SDN Ethernet switches, which creates a tuple that assigns optical and Ethernet ports. Finally, three Brazilian universities (UFRGS, UFMG, and UFES) host computing servers, Universal Software Radio Peripherals (USRPs), and IoT nodes/sensors, providing diverse possibilities for wireless experimental setups. Network topology and resources offered by each testbed are available on FUTEBOL's website.

The Virtualization Layer decouples the software from the underlying hardware by abstracting physical resources to provide the desired virtualized resources. This layer supports Experimentation Slice Virtualization and Abstraction using open source tools, such as Libvirt, OpenVSwitch, OpenStack, OpenFlow, and LXD. The level of abstraction of resources provided to experimenters depends on the type of resource being added to the experiment slice. For example, an experimenter who needs to deploy high-level network services may use LXD containers that enable live migration of these services between different testbeds, while a user who desires to add an optical network to an experiment will receive access to a physical optical port. The testbed resources described in the Converged Physical Infrastructural and the Virtualization Layers are exposed to experimenters through SFA components, and when allocated and provisioned, form an experiment slice. After provisioning, the experimenter can modify optical, wireless, and cloud parameters dynamically throughout the experiment.

The Experiment Control and Orchestration Layer allows the integrated manipulation of optical, wireless, and cloud resources based on three tools: Application-Based Network Orchestrator (ABNO) supports convergent network orchestration by interacting with the network controllers in the wireless and optical domains. Container Orchestration and Provisioning Architecture (COPA) is an orchestration tool for containers, enabling monitoring and live migration of VNFs across remote testbeds. O2CMF provides cloud and NFV orchestration capabilities, such as automatic scaling of VNFs based on predefined policies and monitoring of resource usage.

The ABNO architecture [10] is based on IETF RFC7941 and enables network automation and programmability. The main motivation for developing $\mathrm{ABNO}$ as the network orchestrator in our Control Framework is because most existing SDN controllers rely on monolithic software, offering insufficient flexibility for heterogeneous networks. This inflexibility has led to problems for network operators, such as vendor lockin, lack of support for non-OpenFlow networks, and lack of support for end-to-end multi-domain networks. ABNO offers a flexible solution for both independent and joint optical/wireless orchestration.

COPA is a new orchestration and provisioning tool for containers, which we created to facilitate the migration and monitoring of virtual functions across testbeds. Live migration of traditional Virtual Machines (VMs) is only possible if the testbeds use a common hypervisor. However, we do not wish to force all testbeds to use the same hypervisor on their physical servers; moreover, testbeds are already using different hypervisors for legacy reasons. By using containers to implement virtual functions, an experimenter can use COPA to easily migrate virtual functions across testbeds, regardless of their underlying hypervisor, and with the additional benefit of reducing the virtualization overhead in comparison to a fullfledged VMs. An in-depth description of COPA is available in FUTEBOL's website.

$\mathrm{O} 2 \mathrm{CMF}[11]$ is based on OpenStack and is responsible for creating and managing VNFs in an experiment slice. It comprises an AM and an orchestrator. The latter is offered to the user as a VM of an orchestrator image. This orchestrator supports specifying an NFV experiment with monitoring and scaling policies using the TOSCA language to issue commands and to access any VNF in the experiment. O2CMF vertical scaling, which is based on Libvirt, allows real-time adaptation for provisioned cloud infrastructure, with no unavailability nor restarting of the scaled VM throughout the process. 
Network functions and applications can be developed by using the Service Layer implemented in the FUTEBOL Control Framework architecture. This layer is responsible for deploying, for instance, SDR controllers (e.g., Open Air Interface Evolved Packet Core nodes, Software Radio Systems (SRS) Long Term Evolution (LTE) eNodeB implementation). Moreover, this layer is composed of the user's applications, e.g., a video server that streams video during the experiment. This layer can also provide experimentation tools to generate traffic and monitor the experiment's network activity.

The Testbed Management Layer acts as a mediator between successive requests from Fed4FIRE, interacting with the management components of physical resources. Testbed management also provides a service catalog (i.e., a set of VNFs, services, and tools) to support an experiment. This layer directly accesses the database that stores information about the reserved resources, which is used to provision an experiment slice (i.e., a set of testbed resources that are allocated to an experimenter for a given time). AMs are responsible for dealing with SFA calls, so that users can reserve resources across the federated testbeds using a standard set of credentials. The FUTEBOL Control Framework thus implements an SFA interface for the provisioning of experiment slices. FUTEBOL adopts a common approach for authentication across all testbeds, relying on Fed4FIRE Authority. Network and experiment slicing are provisioned with no warranties, as disclosed in the terms of service.

The management of testbed resources is supported in the FUTEBOL architecture through three tools: O2CMFAggregate Manager, OpenFlow Aggregate Manager, and CBTM. These tools were developed or extended to fulfill different experimentation requirements. In summary, O2CMFAggregate Manager provisions virtual resources for cloud and NFV; OpenFlow Aggregate Manager provides virtual resources for SDN, packet, and optical experimentation; and CBTM supplies virtual resources for SDR and IoT experimentation. Additionally, inter-testbed connectivity can be provided by two different approaches, using either Virtual Extensible LAN (VXLAN) or Open Exchange Software Suite (OESS).

The combination of all the layers that comprise the FUTEBOL Control Framework provides the experimenter with control over multiple network domains, and from physical infrastructure to service management. In the next section, we present an experiment on automatic service scaling that leverages some of the functionalities offered by our framework.

\section{ORChESTRATING AUTOMATIC SERVICE SCALING}

In this section, we present a sample experiment where an experimenter simultaneously implements optical-wireless slice scaling, from the network infrastructure's perspective (Subsection IV-B) and vertical service scaling in the cloud (Subsection IV-C), using the FUTEBOL Control Framework.

\section{A. Experiment setup}

Fig. 2 depicts the physical and virtual resources allocated from three different testbeds in Brazil (UFRGS), Ireland (TCD), and UK (Bristol). Inter-testbed connectivity is achieved through an overlay network (VLAN tagged). Over this network, we established VXLAN tunnels to encapsulate our experiment's control and data traffic. In this experiment, UFRGS and TCD represent edge computing, while Bristol characterizes a central cloud. As a rough estimate, the time required to provision this experiment slice and the associated Control Framework components is approximately five to ten minutes.

The cloud computing resources in the Bristol testbed can be provisioned on demand. The most processing-intensive parts of the experiment are run from this testbed. UFRGS provides VMs as Access Points managed by a Wi-Fi-enabled SDN controller [12]. TCD provides a PON/LTE-based C-RAN, using VMs and USRPs with a full stack LTE implementation of Base Band Unit (BBU), Remote Radio Head (RRH), and user equipment (UE), as well as a software implementation of the Evolved Packet Core (EPC) from OpenAirInterface [13] managed by a custom SDR controller. These LTE devices are interconnected to a PON fronthaul, composed of Optical Line Termination (OLT) and Optical Network Terminal (ONT). For the sake of brevity, the results reported in this section focus on the Bristol central cloud and TCD edge.

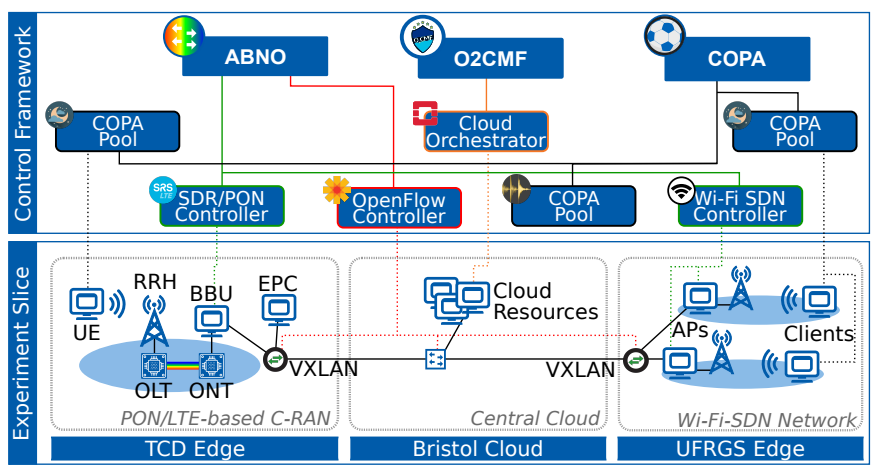

Fig. 2: Experiment components

Fig. 3 shows the workflow of interactions between the Control Framework components (upper part of Fig. 2) to orchestrate the provisioned resources (lower part of Fig. 2) during the execution of the experiment. Initially, COPA is responsible for deploying a distributed service as containers in COPA Pools at cloud and edges, which is achieved in $\sim 5$ seconds. The COPA Pool is a component of COPA that is added to the experiment slice and allows container deployment, monitoring, and migration across testbeds. For this experiment, we utilize a service that analyzes sound samples provided by mobile users worried about their quality of sleep. Users of this service utilize their smartphones (i.e. UEs) to record the ambient audio for a night of sleep and send these audio samples to be analyzed by the cloud service, which is done by a machine learning algorithm that identifies patterns consistent with sleep disorders [14].

In this experiment, we emulate demand from clients activating the service over 24 minutes. Demand initially increases from 1 to 50 clients, and then decreases back to 1 , creating a demand fluctuation. As audio samples are sent over the wireless network, traffic demand fluctuates at the edge, requir- 


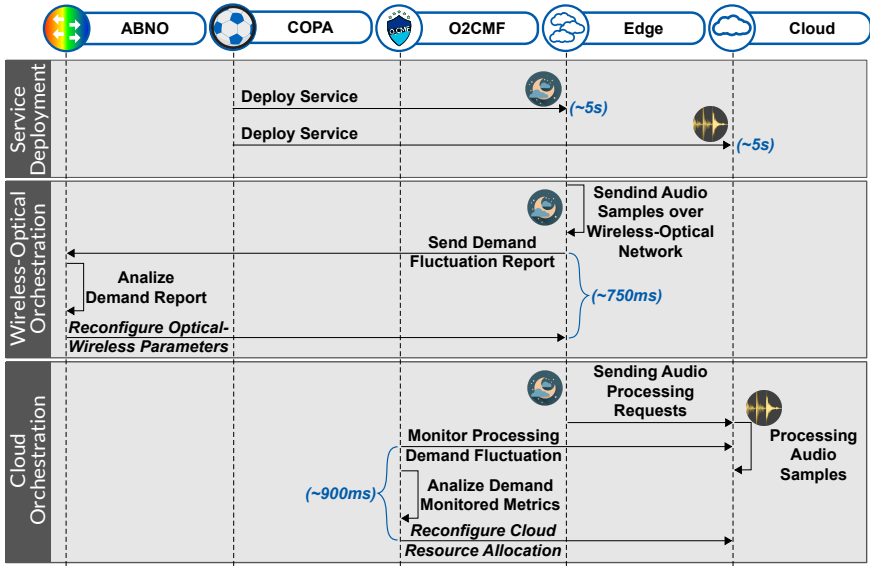

Fig. 3: Experiment components interaction workflow

ing $\mathrm{ABNO}$ to take action and adjust optical-wireless resource allocation. As requests reach the service hosted in the cloud, processing demand also changes, activating the cloud vertical scaling feature provided by the O2CMF orchestrator to adjust the available resources automatically.

\section{B. Scaling optical-wireless resources}

We evaluate the slicing of physical and virtual resources located in the TCD edge, and orchestrated according to the service demand in Bristol's central cloud. A typical scenario might be that of a mobile user on an LTE network requesting dedicated bandwidth through a core and fronthaul network for a pre-determined time. It might otherwise not be feasible to provide fronthaul LTE capacity to this mobile user, were it not for the existence of a converged PON and LTE network close to their location. We have previously demonstrated slicing and scaling of capacity in a converged fixed-mobile network through variable rate LTE over a PON fronthaul [15].

We designed a customized SDR/PON controller for the converged optical and wireless domains that assesses the cell capacity required to provide a particular level of fronthaul application bandwidth, and coordinates in real-time the allocation of this capacity among the BBU, RRH, and PON, achieving $\sim 750 \mathrm{~ms}$ reconfiguration time. This SDR/PON controller provides a RESTful API to connect to ABNO, which specifies optical parameters for provisioning the PON slice. Moreover, $\mathrm{ABNO}$ requests changes in the wireless configuration to the SDR controller, which reconfigures the bandwidth in the cell. LTE cell bandwidth is usually fixed by mobile operators, typically at $20 \mathrm{MHz}$, and requires a fixed transmission of I/Q samples up to a rate of $730 \mathrm{Mbps}$ over a dedicated optical fiber. In our scheme, only the used cell bandwidth needs to be provided, so that the freed-up PON capacity can be re-used by other adjacent LTE cells, or by other lower-priority traffic.

Fig. 4 shows the configuration of the wireless bandwidth and measured dynamics of the maximum rate for cell and fronthaul. The LTE network scaling is shown in response to the fluctuation in the mobile users demand for the service hosted in the cloud. Initially, the bandwidth is set to $1.4 \mathrm{MHz}$, corresponding to the minimal cell bandwidth and minimal fronthaul

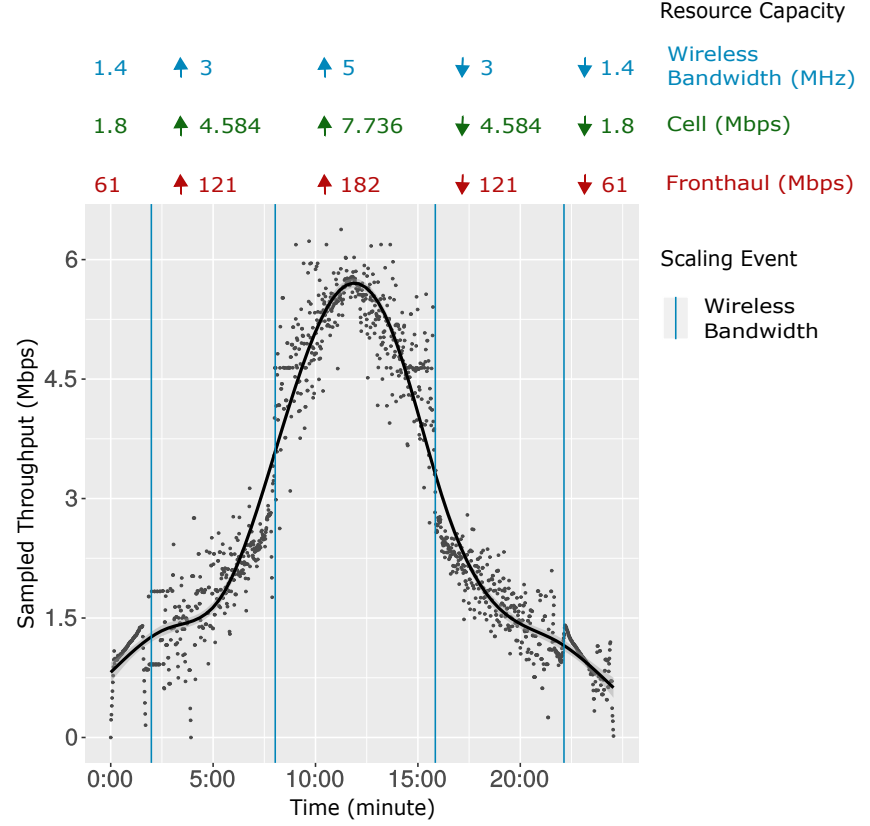

Fig. 4: Orchestration of the physical optical-wireless resources

capacity, since demand is at its minimal ( $1.7 \mathrm{Mbps})$. As more clients activate the service, ABNO dynamically scales the backhaul bandwidth to $3 \mathrm{MHz}$ and $5 \mathrm{MHz}$, achieving a maximum throughput of over $6 \mathrm{Mbps}$. As demand decreases, these changes revert to the initial state. The fluctuation in service demand not only triggers the reconfiguration of the optical-wireless capacity in the edge, but also impacts the demand for the application running in the cloud, as explained next.

\section{Vertical scaling in the cloud}

As the bandwidth capacity increases in the edge, more audio processing requests reach the cloud. O2CMF automatically scales the COPA Pool's resources in response to perceived demand; orchestrated resources are RAM, from 1 to 4 GB, and virtual CPUs, from 1 to 4 . Results for the experiment are presented in Fig. 5, where the time evolution is registered in the lower $\mathrm{x}$-axis, and the clients' demand in the upper $\mathrm{x}$-axis; the y-axis shows the processing time for requests performed. Scaling events, i.e., time instants when O2CMF orchestration increases or decreases the server's resources, are marked with vertical lines, and the status on the allocation of resources is depicted in the upper part of the graph.

As shown in Fig. 5, the mean processing time remains roughly constant from the start of the experiment, with just one client connected, to the eight-minute mark, when 30 clients are served. The following increase in clients from 35 to 50 , observed from the eighth to the eleventh minute, results in a significant increase in the mean processing time. The result indicates that the cloud orchestration is efficient in providing CPU units when demand increases; a decline in performance (i.e., higher response times) is perceived only after the stipulated maximum allocation (4 CPUs) is reached. 


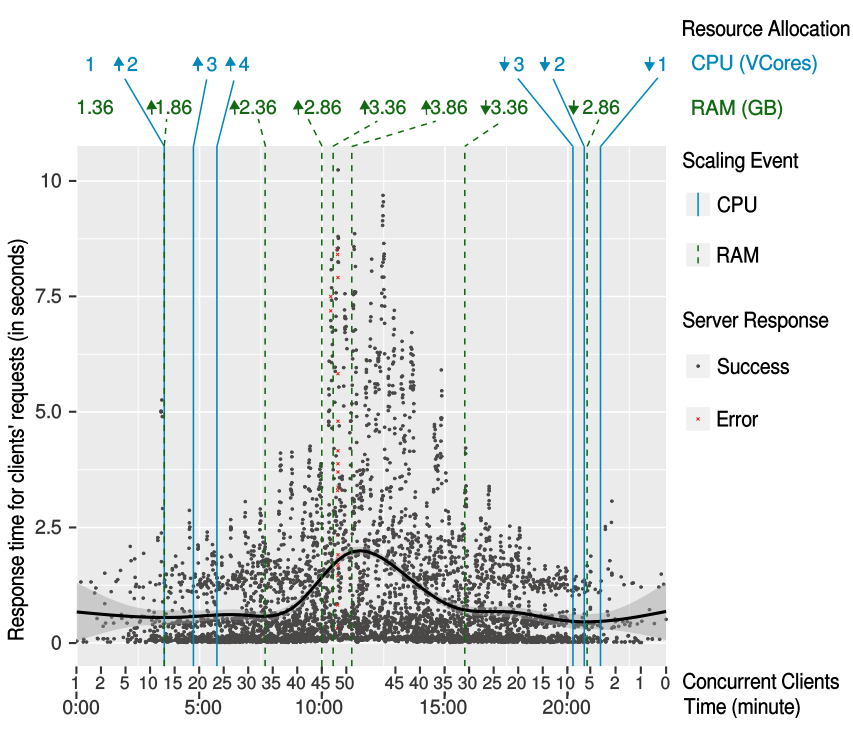

Fig. 5: Cloud orchestration for automatic service scaling

As the experiment uses a memory-intensive service, RAM utilization rapidly increases with the constant influx of new clients, ultimately resulting in service failures (red X's in Fig. 5). RAM insufficiency results in service inability to process incoming requests, which occurs when the scaling is delayed, as seen by the ten-minute mark. Note that all failures occur when demand increases to 45 clients, and not afterwards when it reaches 50. Although the reconfiguration time is fairly small, taking $~ 900 \mathrm{~ms}$ (Fig. 3), this behavior can be explained by the parameters chosen for the orchestration algorithm developed for this experiment, such as the minimal interval between scaling events ( 6 seconds), the usage threshold for scaling resources up ( $80 \%$ for both CPU and RAM), and the fixed amount of resources scaled by each event (0.5 GB RAM; 1 virtual CPU). These parameters indicate an orchestrator tendency to lean towards resource-saving, at the expense of service availability. The experimenter can work the trade-off between resource-saving and service availability by adjusting the parameters with $\mathrm{O} 2 \mathrm{CMF}$.

The experiment investigates the efficient allocation of cloud resources, assisted by the metrics provided by COPA. Throughout the experiment, the average resource allocation for the server was of 2.97 virtual CPUs, and 2.71 GB of RAM. A cloud with no scaling support would require constant allocation of 4 virtual CPUs and $3.86 \mathrm{~GB}$ of RAM. The dynamic allocation represents an average resource-saving of 1.03 virtual CPUs and 1.15 GB of RAM over the the experiment duration. This surplus could thus be reallocated and the resulting resource-saving credited for the underused service, e.g., depending on cloud's service agreement. Orchestration aggressiveness, i.e., how quickly (and by how much) the cloud scales the resources, plays a significant role in this and should be fine-tuned according to each application requirements.

In this experiment, the FUTEBOL Control Framework enabled the network scaling orchestration between the opticalwireless resources to fit the spectrum utilization with the traffic fluctuations from clients activating/deactivating a sample service, and the corresponding vertical scaling of resources in the cloud to cope with the increase/decrease of incoming audio processing requests.

\section{CONCLUSION}

The FUTEBOL Control Framework facilitates experimentation across optical, wireless, and cloud domains, through the slicing of physical resources. To achieve this, we have integrated three tools: O2CMF, developed to allow orchestration for cloud and NFV; ABNO, a convergent network orchestrator that interacts with the network controllers in both optical and wireless domains; and COPA, an orchestration tool for containers to enable the monitoring and live migration of VNFs across testbeds. Moreover, FUTEBOL has established experimental testbeds that offer heterogeneous hardware and software resources for experimentation across optical and wireless networks.

\section{ACKNOWLEDGMENT}

This research received funding from the H2020-BR programme under grant agreement no. 688941. This study was also financed in Brazil by CAPES (Finance Code 001) and FAPES, and by the Science Foundation Ireland under Grant 13/RC/2077.

\section{REFERENCES}

[1] A. Boulogeorgos et al., "Terahertz technologies to deliver optical network quality of experience in wireless systems beyond $5 \mathrm{G}$," IEEE Communications Magazine, vol. 56, no. 6, pp. 144-151, June 2018.

[2] S. Fu et al., "Software defined wireline-wireless cross-networks: Framework, challenges, and prospects," IEEE Communications Magazine, vol. 56, no. 8, pp. 145-151, August 2018.

[3] A. Tzanakaki et al., "Wireless-optical network convergence: Enabling the 5G architecture to support operational and end-user services," IEEE Communications Magazine, vol. 55, no. 10, pp. 184-192, October 2017.

[4] M. Berman et al., "GENI: a federated testbed for innovative network experiments," Computer Networks, vol. 61, pp. 5 - 23, March 2014.

[5] M. Sune et al., "Design and implementation of the OFELIA FP7 facility: the european OpenFlow testbed," Computer Networks, vol. 61, pp. 132150, March 2014.

[6] Futebol project, "Federated Union of Telecommunication Research Facilities for an EU-Brazil Open Laboratory," Accessed on: 2019-04-24. [Online]. Available: http://www.ict-futebol.org.br

[7] T. Huang et al., "A survey on large-scale software defined networking SDN testbeds: Approaches and challenges," IEEE Communications Surveys Tutorials, vol. 19, no. 2, pp. 891-917, November 2017.

[8] P. Marques et al., "Experiments Overview of the EU-Brazil FUTEBOL Project," in European Conference on Networks and Communications, March 2017, pp. 1-2.

[9] T. Rakotoarivelo, M. Ott, G. Jourjon, and I. Seskar, "OMF: a control and management framework for networking testbeds," ACM SIGOPS Operating Systems Review, vol. 43, no. 4, pp. 54-59, January 2010.

[10] A. Hammad et al., "Demonstration of NFV content delivery using SDN-enabled virtual infrastructures," in Optical Fiber Communication Conference, March 2017, pp. 1-2.

[11] I. Ceravolo et al., "O2CMF: Experiment-as-a-service for agile Fed4Fire deployment of programmable NFV," in 2018 Optical Fiber Communications Conference and Exposition (OFC), March 2018, pp. 1-3.

[12] H. Moura et al., "Ethanol: Software defined networking for 802.11 wireless networks," in IFIP/IEEE International Symposium on Integrated Network Management, May 2015, pp. 388-396.

[13] N. Nikaein et al., "OpenAirInterface: an open LTE network in a PC," in Proceedings of the 20th Annual International Conference on Mobile Computing and Networking, ser. MobiCom. ACM, September 2014 pp. 305-308. 
[14] C. F. Bublitz et al., "Unsupervised segmentation and classification of snoring events for mobile health," in IEEE Global Communications Conference, December 2017, pp. 1-6.

[15] P. Alvarez et al., "Experimental demonstration of SDN-controlled variable-rate fronthaul for converged LTE-over-PON," in Optical Fiber Communications Conference and Exposition. IEEE, March 2018, pp. $1-3$.

\section{BIOGRAPHIES}

Cristiano Both is an associate professor of the Applied Computing Graduate Program at the University of Vale do Rio dos Sinos (UNISINOS), Brazil. He is an investigator on research projects funded by H2020 EU-Brazil, CNPq, FAPERGS, and RNP.

Rafael Guimaraes is a Ph.D. student in computer science at UFES and associate professor at Federal Institute of Espirito Santo. His research interests include SDN and cross-layer orchestration/optimization.

Frank Slyne is a post-doctoral researcher at CONNECT/TCD, working on virtualization and multi-tenancy of the metro-access network through the application of SDN and NFV.

Juliano Wickboldt is a professor at UFRGS in Brazil. He has participated as a researcher in several projects funded by Brazilian as well as European agencies. His interests include SDN and 5G technologies.

Magnos Martinello is a professor in the Department of Informatics at UFES in Brazil. His interests include SDN, NFV, and Performance Analysis.

Cristina Dominicini is a Ph.D. candidate at UFES and a professor at Federal Institute of Espírito Santo, Brazil. Her research interests include SDN and NFV.

Rafael Martins is an M.Sc. student at UFRGS, Brazil, where he received his B.Sc. degree in Computer Engineering, in 2018. His research interests include performance analysis of softwarized networks.

Yi Zhang is a postdoctoral research fellow at CONNECT/TCD. $\mathrm{He}$ is a senior member of IEEE. His research is on SDR and wireless networks.

Diego Cardoso is an M.Sc. student at UFES, Brazil, where he also received his B.Sc. degree in Computer Engineering, in 2015.

Rodolfo Villaca is a professor at UFES, Brazil. His interests include SDN, NFV, and Performance Analysis.

Isabella Ceravolo is an M.Sc. student at UFES, Brazil. She received her B.Sc. degree in Information Systems in 2016.

Reza Nejabati is a professor of Intelligent Networks in HighPerformance Research Group in UNIVBRIS. He received the IEEE Charles Kao Award in 2016. His research has contributed to more than 200 peer-reviewed papers and 3 standardization documents.

Johann Marquez-Barja is a professor at University of Antwerp/imec, Belgium, leading the wireless team at IDLab-UAntwerp. He is a member of ACM, IEEE Communications Society, and the IEEE Education Society.

Marco Ruffini is a professor in Computer Science and Statistics at TCD, working on optical network architectures. He is a principal investigator of CONNECT, the Science Foundation Ireland Research Centre in Future Networks and Communications.

Luiz DaSilva holds the chair of Telecommunications at TCD, where he is the director of CONNECT. He is an IEEE Fellow for contributions to resource management in wireless networks. 